\title{
Clearing the path for direct delivery
}

DOI:

10.1038/nri1969
A novel mechanism used by cytotoxic T lymphocytes (CTLs) to deliver lytic granules to the immunological synapse (in this case, a distinct region formed at the contact site of a CTL and a target cell) has recently been described. This mechanism involves the movement of the centrosome - the main microtubule-organizing centre of animal cells - to the immunological synapse, where it transiently comes into contact with the plasma membrane, allowing lytic granules to be delivered directly to the plasma membrane for targeted secretion.

Lytic-granule secretion occurs in the secretory domain of the immunological synapse, which lies next to the central supramolecular activation cluster (cSMAC), but how the lytic granules are delivered to this domain has not been determined until now. Because mobilization of the actin cytoskeleton is required for formation of the immunological synapse, the authors examined the role of actin in lytic-granule secretion. Analysis of actin fused to green fluorescent protein showed that actin was completely cleared from the immunological synapse after conjugation of the CTL with the target cell, indicating that actin is not directly required for lytic-granule secretion.

Stinchcombe et al. then examined the precise position of the centrosome during conjugation by using $\gamma$-tubulin-specific antibody, which specifically labels the centrosome. They showed that the centrosome polarizes towards the immunological synapse and transiently comes into contact with the plasma membrane. Interestingly, the centrosome always localizes to the same site within the immunological synapse, at the edge of the cSMAC. After polarization, the centrioles (which are the microtubules that organize the centrosome itself) are surrounded by lytic granules, and these granules dock with the plasma membrane at the site of centrosome contact.

In addition to actin, IQ-motifcontaining GTPase-activating protein 1 (IQGAP1; a protein that is involved in linking the plus ends of microtubules to actin) is also cleared from the immunological synapse before the centrosome moves to the synapse. The authors suggest that this clearance drives centrosome movement by exerting forces on the microtubule network, which then pulls the centrosome towards the plasma membrane.

The data therefore indicate that, in CTLs, the delivery of lytic granules to the immunological synapse is controlled by the direct movement of the centrosome to the plasma membrane, allowing precise targeting of infected cells by CTLs.

Olive Leavy

ORIGINAL RESEARCH PAPER Stinchcombe, J.

C., Majorovits, E., Bossi, G., Fuller, S. \& Griffiths, G. M. Centrosome polarization delivers secretory granules to the immunological synapse. Nature 443, 462-465 (2006) 